Pacific Journal of Mathematic 


\section{INITIAL SEGMENTS OF ONE-ONE DEGREES}

\section{A. H. LACHLAN}

Let 0 be the one-one degree consisting of the infinite recursive sets whose complements are also infinite. In this paper are studied initial segments of the one-one degrees $\geqq 0$. A characterization is stated of the order types of those initial segments with greatest member which are at the same time initial segments of the many-one degrees. It is shown that any finite initial segment with greatest member is a lattice, and that any finite recursively enumerable initial segment with greatest member is a distributive lattice.

According to the usual definition of one-one reducibility a set of natural numbers $A$ is one-one reducible to a set of natural numbers $B$ written $A \leqq_{1} B$ if there is a one-one recursive function $\rho$ such that $\rho^{-1}(B)=A$. The relation of one-one equivalence $\equiv_{1}$ defined by

$$
A \equiv_{1} B \Leftrightarrow\left[A \leqq_{1} B \text { and } B \leqq_{1} A\right]
$$

is an equivalence relation, and the equivalence classes into which it partitions the sets of natural numbers are called one-one degrees. The natural ordering of these degrees induced by $\leqq_{1}$ is denoted by $\leqq$. In this paper we shall be wholly concerned with the one-one degrees $\geqq 0$. Notice that if $A$ has one-one degree $\geqq 0$ then neither $A$ nor its complement is immune. In this context it is convenient to adopt a new definition of one-one reducibility: we say that $A$ is one-one reducible to a set of natural numbers $B$, again written $A \leqq_{1} B$, if there exists a one-one partial recursive (p.r.) function $\rho$ such that $\operatorname{dom} \rho$ (the domain of $\rho$ ) and $A \cup \operatorname{dom} \rho$ are both recursive, and such that $\rho^{-1}(B)=$ $A \cap \operatorname{dom} \rho$. On sets whose one-one degrees in the old sense are $\geqq 0$ the new definition of $\leqq_{1}$ is the same as the old. Also, for any set $A$ there is a set $B$ whose one-one degree in the old sense is $\geqq 0$, and such that $A \equiv_{1} B$ in the new sense. Thus adopting this new definition leaves the one-one degrees $\geqq 0$ unchanged, and suppresses the remaining one-one degrees.

We adopt the usual definition of many-one reducibility save that $\varnothing, N$ (the set of all natural numbers) are both by convention in the zero many-one degree consisting of all recursive sets.

The only notations likely to be unfamiliar consist in writing $A \oplus B$ for the set $\{2 x \mid x \in A\} \cup\{2 x+1 \mid x \in B\}, A^{\prime}$ for the complement of $A$, and rng $\rho$ for the range of $\rho$.

1. Common initial segments of the many-one and one-one degrees. In [3] we obtained a characterization of the isomorphism 
types of the initial segments of the many-one degrees. We can sharpen the construction in $\S 2$ of [3] to obtain:

THEOREM 1. An upper semilattice $L$ is isomorphic to a common initial segment of the many-one and one-one degrees with greatest member $\neq 0$ if and only if there are sequences $\left\langle D_{i}\right\rangle$ of finite distributive lattices of cardinality $>1$, and $\left\langle\chi_{i}\right\rangle$ of maps, such that for each $i, \chi_{i}$ is an upper semilattice homomorphism of $D_{i}$ into $D_{i+1}$ preserving 0 and 1 , and such that $L$ is isomorphic to the direct limit of the sequence

$$
D_{0} \stackrel{\chi_{0}}{\longrightarrow} D_{1} \stackrel{\chi_{1}}{\longrightarrow} \cdots \stackrel{\chi_{i-1}}{\longrightarrow} D_{i} \stackrel{\chi_{i}}{\longrightarrow} \cdots .
$$

(By saying that $\chi_{i}$ "preserves 0 and 1 " we mean that $\chi_{i}$ maps the least element, greatest element respectively, of $D_{i}$ into the least element, greatest element respectively, of $D_{i+1}$.)

Clearly if we consider only finite initial segments then the condition of the theorem reduces to: $L$ is a finite distributive lattice of cardinality $>1$. By an argument not unlike Friedberg's maximal set construction one can establish:

THeorem 2. If $L$ is a finite distributive lattice then there is a common recursively enumerable (r.e.) initial segment of the manyone and one-one degrees isomorphic to $L$.

It follows from Theorem 1 that the elementary theory of the partial ordering of one-one degrees is undecidable (for details see [1], $\S 3)$. From Theorem 2 the same is true of the partial ordering of the r.e. one-one degrees, but for this one has to use the fact that the sets of first order sentences true of no distributive lattice, and true of some finite distributive lattice respectively, are recursively inseparable. (Unfortunately, we know no reference for this result.)

2. Finite initial segments of the one-one degrees. In this section we shall show that every finite initial segment of the one-one degrees is a lattice of a certain kind.

The disjoint union $\boldsymbol{a} \oplus \boldsymbol{b}$ of a pair of one-one degrees $\boldsymbol{a}, \boldsymbol{b}$ is defined to be the one-one degree of $A \oplus B$ where $A, B$ are any representatives of $\boldsymbol{a}, \boldsymbol{b}$ respectively. The reader will easily verify that $\boldsymbol{a} \oplus \boldsymbol{b}$ depends only on $\boldsymbol{a}, \boldsymbol{b}$ and that the operation $\oplus$ is associative on one-one degrees. For each one-one degree $\boldsymbol{a}$ and natural number $n$, let $n \boldsymbol{a}$ be 0 if $n=0$, and be 


$$
\frac{a \oplus \boldsymbol{a} \oplus \cdots \oplus \boldsymbol{a}}{n \text { times }}
$$

otherwise. A one-one degree $\boldsymbol{a}$ is called a cylinder just if $2 \boldsymbol{a}=\boldsymbol{a}$; notice that $\boldsymbol{a}$ is a cylinder just if all its representatives are cylinders in the sense of Myhill [5].

A one-one degree $\boldsymbol{a}$ is called decomposable if there exist one-one degrees $\boldsymbol{b}, \boldsymbol{c}$ both $<\boldsymbol{a}$ such that $\boldsymbol{a}=\boldsymbol{b} \oplus \boldsymbol{c}$; otherwise $\boldsymbol{a}$ is called indecomposable.

A finite sequence $\left\{\boldsymbol{a}_{1}, \boldsymbol{a}_{2} \cdots, \boldsymbol{a}_{n}\right\}$ of one-one degrees is called a $c a$ nonical decomposition of a one-one degree $\boldsymbol{a}$ if $\boldsymbol{a}=\boldsymbol{a}_{1} \oplus \boldsymbol{a}_{2} \oplus \cdots \oplus \boldsymbol{a}_{n}$ where each $\boldsymbol{a}_{i}$ is indecomposable and where deleting any of $\boldsymbol{a}_{1}, \boldsymbol{a}_{2}, \cdots, \boldsymbol{a}_{n}$ in the last equation destroys the equality. We shall first show that when canonical decompositions exist they are unique. To this end we prove:

LEMMA 1. If

$$
\boldsymbol{a}_{0} \oplus \boldsymbol{a}_{1} \oplus \cdots \oplus \boldsymbol{a}_{m-1}=\boldsymbol{b}_{0} \oplus \boldsymbol{b}_{1} \oplus \cdots \oplus \boldsymbol{b}_{n-1}
$$

with $m, n$ both $\geqq 1$ then there exist one-one degrees $\left\{\boldsymbol{a}_{i, j} \mid i<m, j<n\right\}$ such that

$$
\begin{aligned}
& \boldsymbol{a}_{i}=\boldsymbol{a}_{i, 0} \oplus \boldsymbol{a}_{i, 1} \oplus \cdots \oplus \boldsymbol{a}_{i, n-1}, i<m ; \\
& b_{j}=\boldsymbol{a}_{0, j} \oplus \boldsymbol{a}_{1, j} \oplus \cdots \oplus \boldsymbol{a}_{m-1, j}, j<n .
\end{aligned}
$$

Proof. For $i<m$ let $A_{i}$ be a representative of $\boldsymbol{a}_{i}$, for $j<n$ let $B_{j}$ be a representative of $\boldsymbol{b}_{j}$. Let

$$
\begin{aligned}
& A=\left\{m x+i \mid x \in A_{i} \text { and } i<m\right\}, \\
& B=\left\{n x+j \mid x \in B_{j} \text { and } j<n\right\}
\end{aligned}
$$

then $A, B$ have one-one degrees

$$
\boldsymbol{a}_{0} \oplus \boldsymbol{a}_{1} \cdots \oplus \boldsymbol{a}_{m-1}, \quad \boldsymbol{b}_{0} \oplus \boldsymbol{b}_{1} \oplus \cdots \oplus \boldsymbol{b}_{n-1}
$$

respectively. By hypothesis these are equal and without loss of generality we may suppose that $A, B$ are one-one equivalent in the old sense. By a result of Myhill $([4], \S 5)$ there is a recursive permutation $\rho$ mapping $A$ onto $B$. For $i<m, j<n$ define

$$
A_{i, j}=\left\{x \mid \rho(m x+i)=j \bmod n \text { and } x \in A_{i}\right\} .
$$

Letting $\boldsymbol{a}_{i, j}$ be the one-one degree of $A_{i, j}$ the lemma follows by inspection.

THEOREM 3. If a one-one degree has a canonical decomposition 
then its canonical decomposition is unique to within a permutation.

Proof. Let $\boldsymbol{a}_{0} \oplus \boldsymbol{a}_{1} \oplus \cdots \oplus \boldsymbol{a}_{m-1}, \boldsymbol{b}_{0} \oplus \boldsymbol{b}_{1} \oplus \cdots \boldsymbol{b}_{n-1}$ be two canonical decompositions of the same one-one degree. Applying Lemma 1 and using the notation above, since $\boldsymbol{a}_{i}$ is indecomposable, there exists $\alpha(i)$ such that $\boldsymbol{a}_{i}=\boldsymbol{a}_{i, \alpha(i)}$. Similarly there exists $\beta(i)$ such that $\boldsymbol{b}_{i}=$ $\boldsymbol{a}_{\beta(i), i}$. As $i$ runs through the numbers $<m, \alpha(i)$ must run through all numbers $<n$; for suppose $\alpha(i)$ is never zero then

$$
\boldsymbol{a}_{0} \oplus \boldsymbol{a}_{1} \oplus \cdots \oplus \boldsymbol{a}_{m-1} \leqq \boldsymbol{b}_{1} \oplus \boldsymbol{b}_{2} \oplus \cdots \oplus \boldsymbol{b}_{n-1}
$$

which contradicts the definition of canonical decomposition. Similarly $\beta(i)$ runs through all numbers $<m$. Hence $m=n$ and $\alpha, \beta$ are both permutations of the numbers $<m$. We claim that $\boldsymbol{a}_{i}=\boldsymbol{b}_{\alpha(i)}$ and $\boldsymbol{b}_{i}=$ $\boldsymbol{a}_{\beta(i)}$ for each $i<m$. It is clear that $\boldsymbol{a}_{i} \leqq \boldsymbol{b}_{\alpha(i)}$ and $\boldsymbol{b}_{i} \leqq \boldsymbol{a}_{\beta(i)}$ for each $i<m$. For reductio ad absurdum suppose that our claim is false then the set

$$
S=\left\{\boldsymbol{a}_{i} \mid \boldsymbol{a}_{i}<\boldsymbol{b}_{\alpha(i)} \text { and } i<m\right\} \cup\left\{\boldsymbol{b}_{i} \mid \boldsymbol{b}_{i}<\boldsymbol{a}_{\beta(i)} \text { and } i<m\right\}
$$

is nonempty, and without loss of generality we may suppose that $\boldsymbol{a}_{k}<\boldsymbol{b}_{\alpha(k)}$ and that $\boldsymbol{a}_{k}$ is a maximal member of $S$. By choice of $k$ we have

$$
\boldsymbol{a}_{k}<\boldsymbol{b}_{\alpha(k)}=\boldsymbol{a}_{\beta \alpha(k)}=\boldsymbol{b}_{\alpha \beta \alpha(k)}=\boldsymbol{a}_{\beta \alpha \beta \alpha(k)}=\cdots \text {. }
$$

But $\beta \alpha$ is a permutation and so one of the suffixes of $\boldsymbol{a}$ in the chain of equalities is equal to $k$. This contradiction completes the proof of the theorem.

Let $\boldsymbol{a}, \boldsymbol{b}$ be one-one degrees then $\boldsymbol{b} / \boldsymbol{a}$ is defined to be $\sup \{n \mid n \boldsymbol{a} \leqq \boldsymbol{b}\}$; $\boldsymbol{b} / \boldsymbol{a}$ may take any of the values $0,1,2, \cdots, \infty$. Notice that for any indecomposable $\boldsymbol{a},(\boldsymbol{b} \oplus \boldsymbol{c}) / \boldsymbol{a}=(\boldsymbol{b} / \boldsymbol{a})+(\boldsymbol{c} / \boldsymbol{a})$ where it is understood that $x+\infty=\infty+x=\infty$. If $\boldsymbol{a}$ is not a cylinder then $\boldsymbol{a}, 2 \boldsymbol{a}, 3 \boldsymbol{a}, \cdots$ is a strictly increasing sequence of one-one degrees whence $a / a=1$. If $a$ is a cylinder then $\boldsymbol{b} / \boldsymbol{a}$ is zero or $\infty$, and if $\boldsymbol{a} \leqq \boldsymbol{b}$ and $\boldsymbol{a}$ is a cylinder then $\boldsymbol{b} \oplus \boldsymbol{a}=\boldsymbol{b}$.

Notice that if a one-one degree $\boldsymbol{a}$ has only finitely many predecessors then some sequence of the degrees $\leqq \boldsymbol{a}$ must constitute a canonical decomposition of $\boldsymbol{a}$; for example if $\boldsymbol{a}$ is indecomposable then it is its own canonical decomposition.

Lemma 2. Let $\boldsymbol{a}, \boldsymbol{b}$ be one-one degrees such that $\boldsymbol{a}$ has only finitely many prodecessors. Let $\boldsymbol{a}_{0} \oplus \boldsymbol{a}_{1} \oplus \cdots \oplus a_{m}$ be the canonical decomposition of $\boldsymbol{a}$. Then $\boldsymbol{a} \leqq \boldsymbol{b}$ if and only if $\boldsymbol{a} / \boldsymbol{a}_{i}$ for all $i \leqq m$.

Proof. The "only if" part is immediate. For the "if" part we 
proceed by induction on $m$. Since the result is immediate for $m=0$ we may suppose that $m>0$. Let $\boldsymbol{a}^{*}=\boldsymbol{a}_{0} \oplus \boldsymbol{a}_{1} \oplus \cdots \boldsymbol{a}_{m-1}$ and suppose that $\boldsymbol{a} / \boldsymbol{a}_{i} \leqq \boldsymbol{b} / \boldsymbol{a}_{i}$ for all $i \leqq m$. It follows from our induction hypothesis that $\boldsymbol{a}^{*} \leqq \boldsymbol{b}$ since it is easy to see that $\boldsymbol{a}_{0} \oplus \boldsymbol{a}_{1} \oplus \cdots \oplus \boldsymbol{a}_{m-1}$ is the canonical decomposition of $\boldsymbol{a}^{*}$. Without loss of generality we assume that $\boldsymbol{a}_{m}$ is minimal among $\boldsymbol{a}_{0}, \boldsymbol{a}_{1}, \cdots, \boldsymbol{a}_{m}$.

Let $B$ be a representative of $\boldsymbol{b}$. It is convenient to introduce a map $\Psi$ from the r.e. sets onto the one-one degrees $\leqq b$. If $W$ is finite let $\Psi(W)=\mathbf{0}$, if $W$ is an infinite r.e. set let $\omega$ be any one-one recursive function whose range is $W$ and let $\Psi(W)$ be the one-one degree of $\{x \mid \omega(x) \in B\}$. It is easy to see that $\Psi(W)$ does not depend upon the choice of $w$, that $\Psi$ is order preserving, and that

$$
\Psi(W)=\boldsymbol{w}_{0} \oplus \boldsymbol{w}_{1} \oplus \cdots \oplus \boldsymbol{w}_{e-1}
$$

if and only if $W$ is the union of disjoint r.e. sets $W_{0}, W_{1}, \cdots, W_{e-1}$ such that $\Psi\left(W_{i}\right)=\boldsymbol{w}_{i}$ for each $i<e$. Since $\boldsymbol{a}^{*} \leqq \boldsymbol{b}$ there exist disjoint r.e. sets $A_{0}, A_{1}, \cdots, A_{m-1}$ such that $\Psi\left(A_{i}\right)=\boldsymbol{a}_{i}$ for each $i<m$. There are two cases to consider:

Case 1. $\boldsymbol{a} / \boldsymbol{a}_{m}$ is finite. Then $\boldsymbol{a}_{m}$ is not a cylinder because of the remarks that precede this lemma. Let $\boldsymbol{a}^{*} / \boldsymbol{a}_{m}=k$ so that $\boldsymbol{b} / \boldsymbol{a}_{m} \geqq \boldsymbol{a} / \boldsymbol{a}_{m} \geqq$ $k+1$. It easily follows that there are disjoint r.e. sets $C_{0}, C_{1}, \cdots$, $C_{k}$ such that $\Psi\left(C_{j}\right)=\boldsymbol{a}_{m}$ for each $j \leqq k$. Effectively enumerate the r.e. sets $A_{0}, A_{1}, \cdots, A_{m-1}, C_{0}, C_{1}, \cdots, C_{k}$ simultaneously stage by stage letting $A_{i}^{s}$ and $C_{i}^{s}$ be the finite sets enumerated up to stage $s$ for each $i<m$ and $j \leqq k$. We then define

$$
\begin{aligned}
& F_{i}=\left\{x \mid(\exists s)\left[x \in A_{i}^{s} \quad \text { and } \quad(\forall j)_{j \leqq k}(\forall r)_{r<s}\left[x \notin C_{j}^{r}\right]\right]\right\}, \quad i<m ; \\
& G_{j}=\left\{x \mid(\exists s)\left[x \in C_{j}^{s} \quad \text { and } \quad(\forall i)_{i<m}(\forall r)_{r \leqq s}\left[x \notin A_{j}^{r}\right]\right]\right\}, \quad j \leqq k \text {. }
\end{aligned}
$$

Also, for each $i<m$ let $F_{i}^{*}$ be the r.e. set $A_{i}-F_{i}$, and for each $j \leqq k$ let $G_{j}^{*}$ be the r.e. set $C_{j}-G_{j}$. Observe that $F_{i}^{*} \leqq G_{0} \cup G_{1} \cup \cdots \cup G_{k}$ for all $i<m$ and $G_{j}^{*} \subseteq F_{0} \cup F_{1} \cup \cdots \cup F_{m-1}$ for all $j \leqq k$. Also, it can be seen that $F_{0}, F_{1}, \cdots, F_{m-1}, G_{0}, G_{1}, \cdots, G_{k}$ constitute a decomposition of $A_{0} \cup A_{1} \cup \cdots \cup A_{m-1} \cup C_{0} \cup C_{1} \cup \cdots \cup C_{k}$ into disjoint r.e. sets.

For each $i \leqq m, \boldsymbol{a}_{i}$ is indecomposable. This implies that either $\Psi\left(F_{i}\right)=\boldsymbol{a}_{i}$ or $\Psi\left(F_{i}^{*}\right)=\boldsymbol{a}_{i}$ for each $i<m$ and that either $\Psi\left(G_{j}\right)=\boldsymbol{a}_{m}$ or $\Psi\left(G_{j}^{*}\right)=\boldsymbol{a}_{m}$ for each $j \leqq k$. Define $P, Q$ by

$$
\begin{aligned}
& P=\left\{i \mid i<m \text { and } \Psi\left(F_{i}\right)=\boldsymbol{a}_{i}\right\}, \\
& Q=\{i \mid i<m \text { and } i \notin P\} .
\end{aligned}
$$

Now if $i \in Q$ then $\Psi\left(F_{i}^{*}\right)=\boldsymbol{a}_{i}$. But $F_{i}^{*}$ is the union of the disjoint r.e. sets $F_{i}^{*} \cap G_{0}, \cdots, F_{i}^{*} \cap G_{k}$ so that for each $i \in Q$ there exists $\alpha(i) \leqq k$ 
such that $\Psi\left(F_{i}^{*} \cap G_{\alpha(i)}\right)=\boldsymbol{a}_{i}$.

Consider the set

$$
D=\alpha(Q) \cup\left\{j \mid j<k \text { and } \Psi\left(G_{j}^{*}\right)=\boldsymbol{a}_{m}\right\},
$$

and let $\beta$ be a map from $\alpha(Q)$ into $Q$ such that $\alpha \beta(j)=j$ for all $j \in \alpha(Q)$. For each $j \in D$ we define

$$
D_{j}=\left\{\begin{array}{l}
G_{j}^{*} \text { if } \Psi\left(G_{j}^{*}\right)=\boldsymbol{a}_{m}, \\
F_{\beta(j)}^{*} \cap G_{j} \text { otherwise } .
\end{array}\right.
$$

This is a sound definition because if $\Psi\left(G_{j}^{*}\right) \neq \boldsymbol{a}_{m}$ then $j \in \alpha(Q)$ and so $\beta(j)$ is defined.

We first claim that $\Psi\left(D_{j}\right)=\boldsymbol{a}_{m}$ for all $j \in D$. For, if $\Psi\left(G_{j}^{*}\right) \neq \boldsymbol{a}_{m}$ then $\beta(j)$ is defined and $\Psi\left(G_{j}\right)=\boldsymbol{a}_{m}$. Since $\Psi$ is order preserving it follows that

$$
\boldsymbol{a}_{\beta(j)}=\Psi\left(F_{\beta(j)}^{*} \cap G_{j}\right) \leqq \Psi\left(G_{j}\right)=\boldsymbol{a}_{m},
$$

and because $\boldsymbol{a}_{m}$ is minimal we deduce that $\boldsymbol{a}_{\beta(j)}=\boldsymbol{a}_{m}$. It follows that $\Psi\left(F_{\beta(j)}^{*} \cap G_{j}\right)=\Psi\left(D_{j}\right)=\boldsymbol{a}_{m}$. This proves our first claim.

Next we use this to deduce that $j \notin D$ for some $j \leqq k$. For, otherwise $(k+1) \boldsymbol{a}_{m}=\Psi\left(D_{0} \cup \cdots \cup D_{k}\right) \leqq \Psi\left(A_{0} \cup \cdots \cup A_{m-1}\right)=\boldsymbol{a}^{*}$, because $D_{0}, D_{1}, \cdots, D_{k}$ are disjoint r.e. subset of $A_{0} \cup \cdots \cup A_{m-1}$. But $\boldsymbol{a}^{*} / \boldsymbol{a}_{m}=k$ and so this is impossible.

Now let $j$ be a fixed number $\leqq k$ which does not belong to $D$ and for each $i \leqq m$ define

$$
E_{i}=\left\{\begin{array}{l}
F_{i} \text { if } i \in P \\
F_{i}^{*} \cap G_{\alpha(i)} \text { if } i \in Q, \\
G_{j} \text { if } i=m .
\end{array}\right.
$$

Then $E_{0}, E_{1}, \cdots, E_{m}$ are disjoint r.e. sets and $\Psi\left(E_{i}\right)=\boldsymbol{a}_{i}$ for all $i \leqq m$. Since $\Psi\left(E_{0} \cup \cdots \cup E_{m}\right)=\boldsymbol{a}$ it follows that $\boldsymbol{a} \leqq \boldsymbol{b}$ which completes this case.

Case 2. $\quad \boldsymbol{a} / \boldsymbol{a}_{m}=\infty$. Then $\boldsymbol{a}_{m}$ is cylinder, otherwise $\boldsymbol{a}$ would have an infinite number of predecessors. Suppose $\boldsymbol{a}_{m} \leqq \boldsymbol{a}^{*}$, then by the remarks preceding the lemma we have $\boldsymbol{a}=\boldsymbol{a}^{*} \oplus \boldsymbol{a}_{m}=\boldsymbol{a}^{*}$ which contradicts $\boldsymbol{a}_{0} \oplus \boldsymbol{a}_{1} \oplus \cdots \oplus \boldsymbol{a}_{m}$ being a canonical decomposition of $\boldsymbol{a}$. Thus $\boldsymbol{a}^{*} / \boldsymbol{a}_{m}=\mathbf{0}$. Now, taking $k=0$, proceed just as in the last case.

Before turning to the next theorem, we note that any finite upper semilattice is in fact a lattice as long as it has a least element. For, if least upper bounds exist then greatest lower bounds exist by the definition: 


$$
\boldsymbol{b} \cap \boldsymbol{c}=\sup \{\boldsymbol{x} \mid \boldsymbol{x} \leqq \boldsymbol{b} \text { and } \boldsymbol{x} \leqq \boldsymbol{c}\}
$$

THEOREM 4. Let a be a one-one degree which has only a finite number of predecessors then the predecessors of a form a lattice.

Proof. In view of the observation that precedes the theorem, we have only to show that the predecessors of $\boldsymbol{a}$ form an upper semilattice. Let $\boldsymbol{b}, \boldsymbol{c}$ be any one-one degrees $\leqq \boldsymbol{a}$ and suppose that $\boldsymbol{a}$ has only a finite number of predecessors. Let $\boldsymbol{a}_{1}, \boldsymbol{a}_{2}, \cdots, \boldsymbol{a}_{p}$ be an enumeration of all the indecomposable one-one degrees $\leqq \boldsymbol{a}$ arranged so that for any $i, j$

$$
p \geqq i>j \geqq 1 \text { implies } \boldsymbol{a}_{i} \geqq \boldsymbol{a}_{j} \text {. }
$$

To show that $\boldsymbol{b} \cup \boldsymbol{c}$ exists we shall define an increasing sequence $\boldsymbol{e}_{1}, \boldsymbol{e}_{2}, \cdots, \boldsymbol{e}_{p}$ of one-one degrees $\leqq \boldsymbol{a}$ and simultaneously we shall show that for all $i, 1 \leqq i \leqq p$, and all $\boldsymbol{d} \leqq \boldsymbol{a}$

$$
b \leqq d \quad \text { and } \quad c \leqq d . \rightarrow e_{i} \leqq d
$$

and

$$
\boldsymbol{e}_{i} / \boldsymbol{a}_{i} \geqq \max \left(\boldsymbol{b} / \boldsymbol{a}_{i}, \boldsymbol{c} / \boldsymbol{a}_{i}\right)
$$

Define

$$
\boldsymbol{e}_{1}=\left\{\begin{array}{l}
\boldsymbol{a}_{1} \text { if one of } \boldsymbol{b} / \boldsymbol{a}_{1}, \boldsymbol{c} / \boldsymbol{a}_{1} \text { is } \infty \\
\max \left(\boldsymbol{b} / \boldsymbol{a}_{1}, \boldsymbol{c} / \boldsymbol{a}_{1}\right) \boldsymbol{a}_{1} \text { otherwise }
\end{array}\right.
$$

then $e_{1} \leqq \boldsymbol{a}$ and (1), (2) are certainly satisfied for $i=1$. Suppose that $\boldsymbol{e}_{i}$ has been defined for $1 \leqq i \leqq k$ where $k<p$ so that (1), (2) are satisfied for $1 \leqq i \leqq k$. If

$$
\boldsymbol{e}_{k} / \boldsymbol{a}_{k+1} \geqq \max \left(\boldsymbol{b} / \boldsymbol{a}_{k+1}, \boldsymbol{c} / \boldsymbol{a}_{k+1}\right),
$$

then set $e_{k+1}=e_{k}$, in which case (1), (2) are clearly satisfied for $i=$ $k+1$. Otherwise let $r, s$ be the numbers on the left, right respectively of the last inequality with the proviso that if $\boldsymbol{a}_{k+1}$ is a cylinder then $s=1$. Set $\boldsymbol{e}_{k+1}=\boldsymbol{e}_{k} \oplus(s-r) \boldsymbol{a}_{k+1}$. Any degree appearing in the canonical decomposition of $\boldsymbol{e}_{k+1}$ is clearly one of $\boldsymbol{a}_{1}, \boldsymbol{a}_{2}, \cdots, \boldsymbol{a}_{k+1}$. Hence by Lemma $2, \boldsymbol{e}_{k+1} \leqq \boldsymbol{a}$. Suppose that $\boldsymbol{b} \leqq \boldsymbol{d}$ and $\boldsymbol{c} \leqq \boldsymbol{d}$ then from (1) for $i=k$ we know that $\boldsymbol{e}_{k} \leqq \boldsymbol{d}$ which implies that $\boldsymbol{d} / \boldsymbol{a}_{i} \geqq \boldsymbol{e}_{k} / \boldsymbol{a}_{i}$ for $1 \leqq i \leqq k$. But since we are assuming $\boldsymbol{e}_{k+1} \neq \boldsymbol{e}_{k}$ we can deduce that $\boldsymbol{a}_{k+1}$ is different from all of $\boldsymbol{a}_{1}, \boldsymbol{a}_{2}, \cdots, \boldsymbol{a}_{k}$ and does not exceed any of them. Thus

$$
\boldsymbol{d} / \boldsymbol{a}_{i} \geqq \boldsymbol{e}_{k} / \boldsymbol{a}_{i}=\boldsymbol{e}_{k+1} / \boldsymbol{a}_{i}, \quad 1 \leqq i \leqq k
$$


Also

$$
\boldsymbol{d} / \boldsymbol{a}_{k+1} \geqq s=\boldsymbol{e}_{k+1} / \boldsymbol{a}_{k+1} \text {. }
$$

Any degree which appears in the canonical decomposition of $\boldsymbol{e}_{k+1}$ is one of $\boldsymbol{a}_{1}, \boldsymbol{a}_{2}, \cdots, \boldsymbol{a}_{k+1}$. Hence $\boldsymbol{e}_{k+1} \leqq \boldsymbol{d}$ by Lemma 2. It is immediate that (2) is satisfied for $i=k+1$. This completes the induction step. It is clear that $\boldsymbol{e}_{p}$ is the least upper bound of $\boldsymbol{b}, \boldsymbol{c}$. For $\boldsymbol{b} \leqq \boldsymbol{e}_{p}$ and $c \leqq e_{p}$ by (2) and Lemma 2, and $e_{p}$ is the least among the common upper bounds of $\boldsymbol{b}, \boldsymbol{c}$ from (1). This completes the proof of the theorem.

The next theorem gives a necessary condition for a finite lattice to be an initial segment of one-one degrees.

Theorem 5. Let a be a one-one degree with only a finite number of predecessors then there exist finite separated distributive lattices $L_{1}, L_{2}$ and an order preserving map $\Psi$ of $L_{2}$ onto the one-one degrees $\leqq \boldsymbol{a}$ satisfying the following four conditions in which $\subseteq$, $\varnothing$ denote the partial order and least element of $L_{2}$ respectively.

(i) $L_{1}$ is an initial segment of $L_{2}$ containing at least $\varnothing$ and $\Psi\left(L_{1}\right)$ is a distributive lattice,

(ii) if $x \cap y=\varnothing$ then $\Psi(x \cup y)=\Psi(x)$ if and only if $y \in L_{1}$ and $\Psi(y) \leqq \Psi(x)$,

(iii) if $x_{1} \cap x_{2}=y_{1} \cap y_{2}=\varnothing$ and $\Psi\left(x_{i}\right)=\Psi\left(y_{i}\right)$ for $i=1,2$ then $\Psi\left(x_{1} \cup x_{2}\right)=\Psi\left(y_{1} \cup y_{2}\right)$,

(iv) for any $x \in L_{2}$ and $\boldsymbol{y} \leqq \Psi(x)$ there exists $y \leqq x$ such that $\Psi(y)=\boldsymbol{y}$.

Proof. We proceed by induction on the number of predecessors of $\boldsymbol{a}$. When $\boldsymbol{a}=\mathbf{0}$ the theorem is obvious. Suppose that $\boldsymbol{b}>\mathbf{0}$ has only a finite number of predecessors and assume the result for $\boldsymbol{a}<\boldsymbol{b}$. Assume in addition that for any $\boldsymbol{a}<\boldsymbol{b}$ we can choose $L_{1}, L_{2}, \Psi$ such that $\Psi\left(L_{1}\right)$ consists of the cylinders $\leqq \boldsymbol{a}$, and if $x_{1} \cap x_{2}=\varnothing$ then $\Psi\left(x_{1} \cup x_{2}\right)=\Psi\left(x_{1}\right) \oplus \Psi\left(x_{2}\right)$.

Case 1. Suppose that $\boldsymbol{b}$ has just one maximal predecessor $\boldsymbol{b}_{1}$. Let $L_{1}^{(1)}, L_{1}^{(1)}, \Psi^{(1)}$ be the finite separated distributive lattices and map whose existence is predicted by the theorem, and satisfying the additional conditions, when $\boldsymbol{a}=\boldsymbol{b}_{1}$. Let $L_{2}=L_{2}^{(1)} \cup\{b\}$ where $b \notin L_{2}^{(1)}$, and let

$$
L_{1}=\left\{\begin{array}{l}
L_{1}^{(1)} \cup\{b\} \text { if } \boldsymbol{b} \text { is a cylinder } \\
L_{1}^{(1)} \text { otherwise. }
\end{array}\right.
$$

Let $x \leqq y$ in $L_{2}$ if and only if $y=b$ or $x, y$ are both in $L_{2}^{(1)}$ and $x \subseteq y$ in $L_{2}^{(1)}$. Let $\Psi(b)=\boldsymbol{b}$ and otherwise let $\Psi$ agree with $\Psi^{(1)}$. It is easy 
to check (i) - (iv) and the additional conditions for $\boldsymbol{a}=\boldsymbol{b}$.

Case 2. Suppose that $\boldsymbol{b}$ has at least two maximal predecessors $\boldsymbol{b}_{1}, \boldsymbol{b}_{2}$. Using the technique of Lemma 2 it is easy to show that there exist $\boldsymbol{c}_{1} \leqq \boldsymbol{b}_{1}$ and $\boldsymbol{c}_{2} \leqq \boldsymbol{b}_{2}$ such that $\boldsymbol{b}=\boldsymbol{c}_{1} \oplus \boldsymbol{c}_{2}$. For $i=1,2$ let $L_{1}^{(i)}$, $L_{2}^{(i)}, \Psi^{(i)}$ be the finite separated distributive lattices and the map predicted by the theorem, and satisfying the additional conditions, when $\boldsymbol{a}=\boldsymbol{c}_{i}$. For $j=1,2$ let $L_{j}$ be the direct union of $L_{j}^{(1)}, L_{j}^{(2)}$. Then for $j=1,2, L_{j}$ is a finite separated distributive lattice, and $L_{1}$ is an initial segment of $L_{2}$. For any pair $(x, y)$ in $L_{2}$ define

$$
\Psi(x, y)=\Psi^{(1)}(x) \oplus \Psi^{(2)}(y)
$$

It is again easy to check (i)-(iv) and the additional conditions for $\boldsymbol{a}=\boldsymbol{b}$. This completes the proof of the theorem.

We conjecture that the converse of Theorem 5 is true in the following sense. Let $P$ be any partially ordered set such that there exist finite separated distributive lattice $L_{1}, L_{2}$ and an order preserving map $\Psi$ of $L_{2}$ onto $P$ such that (i)-(iv) are satisfied. We conjecture that there exists a one-one degree $\boldsymbol{p}$ such that the lattice of one-one degree $\leqq \boldsymbol{p}$ is isomorphic to $P$, and that under the isomorphism $\Psi\left(L_{1}\right)$ maps into the set of cylinders $\leqq p$, and that for any $x_{1}, x_{2}$ disjoint in $L_{2}$ the image of $\Psi\left(x_{1} \cup x_{2}\right)$ under the isomorphism is the disjoint union of the images of $\Psi\left(x_{1}\right), \Psi\left(x_{2}\right)$. In the next section we shall prove a particular case of this conjecture. Although it is by no means obvious this conjecture is consistent with the earlier results of this section, e.g., it follows from the conditions imposed upon $P$ that $P$ is a lattice. This can be shown by a suitable modification of the proof of the earlier results.

There are a number of observations which are almost immediate from what has been proved above. First notice that if $\boldsymbol{a}_{1}, \boldsymbol{a}_{2}$ both have only a finite number of predecessors then $\boldsymbol{a}_{1} \oplus \boldsymbol{a}_{2}$ has only a finite number of predecessors and so from Theorem 4, $\boldsymbol{a}_{1} \cup \boldsymbol{a}_{2}$ and $\boldsymbol{a}_{1} \cap \boldsymbol{a}_{2}$ exist in the partial ordering of one-one degrees. Next, if $\boldsymbol{a}_{1}$, $\boldsymbol{a}_{2}, \cdots, \boldsymbol{a}_{n}$ are distinct one-one degrees minimal $>\mathbf{0}$, then

$$
\boldsymbol{a}_{1} \cup \boldsymbol{a}_{2} \cup \cdots \cup \boldsymbol{a}_{n}=\boldsymbol{a}_{1} \oplus \boldsymbol{a}_{2} \oplus \cdots \oplus \boldsymbol{a}_{n}
$$

and the one-one degrees $\leqq \boldsymbol{a}_{1} \oplus \boldsymbol{a}_{2} \oplus \cdots \oplus \boldsymbol{a}_{n}$ form a Boolean algebra of order $2^{n}$. This shows that neither of the two nondistributive lattices of order five can be isomorphic to initial segments of the oneone degrees. One can also show rather easily that many other nondistributive lattices of small order cannot be isomorphic to initial segments of the one-one degrees, e.g., the lattices depicted in the usual way: 


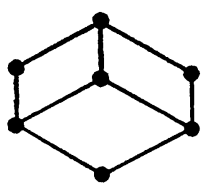

Fig. 1

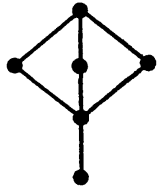

Fig. 2

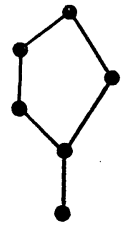

Fig. 3

As an example take the second of these and suppose for reductio and absurdum that the initial segment whose greatest member is $\boldsymbol{a}$ is isomorphic to this lattice. Let $L_{1}, L_{2}, \Psi$ be the separated distributive lattices and the map given by Theorem 5 and let the additional conditions of the proof of Theorem 5 be satisfied. Let $\boldsymbol{a}_{1}, \boldsymbol{a}_{2}, \boldsymbol{a}_{3}$ be maximal below $\boldsymbol{a}$, and let $\boldsymbol{b}$ be minimal above 0 . Let $a_{1}, a_{2}$ be elements of $L_{2}$ such that $\Psi\left(a_{i}\right)=\boldsymbol{a}_{i}$ for $i=1,2$. Since $L_{2}$ is separated there exist $b_{1}, b_{2}$ such that $b_{i} \leqq a_{i}$ for $i=1,2$ and such that $b_{1} \cup b_{2}=a_{1} \cup a_{2}$ while $b_{1} \cap b_{2}=\varnothing$. Clearly, $\Psi\left(b_{i}\right) \leqq \boldsymbol{a}_{i}$ for $i=1,2$. Also,

$$
\Psi\left(a_{1} \cup a_{2}\right)=\Psi\left(b_{1} \cup b_{2}\right)
$$

is $\geqq$ both $\boldsymbol{a}_{1}$ and $\boldsymbol{a}_{2}$ since $\Psi$ is order preserving. Thus $\Psi\left(b_{1} \cup b_{2}\right)=\boldsymbol{a}$. From (iv) there exists $a_{3} \subseteq b_{1} \cup b_{2}$ such that $\Psi\left(a_{3}\right)=\boldsymbol{a}_{3}$. Now for $i=$ $1,2 \Psi\left(a_{3} \cap b_{i}\right)$ is $\leqq$ both $\boldsymbol{a}_{3}$ and $\Psi\left(b_{i}\right)$ and hence is either $\mathbf{0}$ or $\boldsymbol{b}$. But $\Psi\left(a_{3}\right)=\Psi\left(a_{3} \cap b_{1}\right) \oplus \Psi\left(a_{3} \cap b_{2}\right)$, whence $\boldsymbol{a}_{3}=\boldsymbol{b} \oplus \boldsymbol{b}$. Similarly, $\boldsymbol{a}_{1}=\boldsymbol{b} \oplus \boldsymbol{b}$, contradiction.

3. Not every initial segment is distributive. In this section we verify one particular consequence of our conjecture and in so doing we construct an initial segment of one-one degrees which is isomorphic to the lattice:

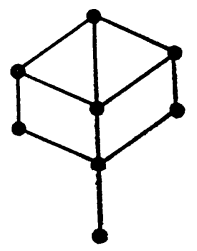

Fig. 4

Let $M$ be a maximal r.e. set. Let $\mathscr{K}, \mathscr{K}(M), \mathscr{L}(M)$ denote the classes of all r.e. sets, all r.e. subsets of $M$, all recursive subsets of $M$ respectively. For our construction we shall require the following:

Lemma 3. There exists a class $\mathscr{C l}$ of r.e. sets, $\mathscr{L}(M) \cong \mathscr{C} \cong$ $\mathscr{K}(M)$, such that if $W$ is a finite union of members of $\mathscr{M}$ then $W \in \mathscr{M}$ and $M-W$ is infinite, and such that if $W \in \mathscr{K}(M)-\mathscr{M}$ then some member of $\mathscr{l l}$ complements $W$ relative to $M$. 
Since this is easy we omit its proof; notice that the property we require of $\mathscr{C}$ is simply that $\mathscr{K}(M)-\mathscr{C}$ should be the intersection with $\mathscr{K}(M)$ of a nonprincipal ultrafilter over $M$ which contains no member of $\mathscr{L}(M)$. It is clear that there exists an increasing sequence $M_{0}, M_{1}, \ldots$ of members of $\mathscr{C}$ such that every member of $\mathscr{C}$ is $\subseteq M_{i}$ for some $i$. Choose a strictly increasing sequence $m_{0}, m_{1}, \cdots$ of members of $M$ such that for all $i, m_{i} \in M-M_{i}$. Since $M-M_{i}$ is infinite for each $i$, the sequence $m_{0}, m_{1}, \cdots$ can be found. Let $M^{*}=$ $\left\{m_{i} \mid i \geqq 0\right\}$, then $M^{*} \cap M_{i}$ is finite for each $i$. For $j=0,1$ let $R_{j}=$ $\{2 x+j \mid x \geqq 0\}$ and $S_{j}=\{2 x+j \mid x \in M\}$. Let $P_{0}, P_{1}$ be infinite disjoint sets both $\subseteq M^{\prime}$. Let $a$ be the one-one degree of the set

$$
A=\left\{2 x+j \mid x \in M^{*} \text { and } j \leqq 1\right\} \cup\left\{2 x+j \mid x \in P_{j} \text { and } j \leqq 1\right\} \text {. }
$$

We shall show that the degrees $\leqq \boldsymbol{a}$ with their natural ordering do form a lattice of order eight isomorphic to the one depicted above. We shall again use the map $\Psi$ introduced in Lemma 2 . If $W$ is finite then $\Psi(W)=\mathbf{0}$, while if $W$ is an infinite r.e. set we let $\omega$ be any one-one recursive function whose range is $W$ and $\Psi(W)$ is defined to be the one-one degree of $\{x \mid \omega(x) \in A\}$. We shall use the properties of $\Psi$ which were stated above without proof. Let $\boldsymbol{a}_{j}$ be $\Psi\left(R_{j}\right)$ for $j=$ 0,1 and let $\boldsymbol{m}=\Psi\left(S_{0}\right)=\Psi\left(S_{1}\right)$; it is apparent that $\Psi\left(S_{0}\right)=\Psi\left(S_{1}\right)$ when one considers the map: $2 x$ goes to $2 x+1$, restricted to $S_{0}$. It is clear that $\boldsymbol{a}=\boldsymbol{a}_{0} \oplus \boldsymbol{a}_{1}$, that $\boldsymbol{m} \leqq \boldsymbol{a}_{j}$ for $j=0,1$. Thus all of $\mathbf{0}, \boldsymbol{m}, \boldsymbol{m} \oplus \boldsymbol{m}$, $\boldsymbol{a}_{0}, \boldsymbol{a}_{1}, \boldsymbol{a}_{0} \oplus \boldsymbol{m}, \boldsymbol{a}_{1} \oplus \boldsymbol{m}, \boldsymbol{a}$ are $\leqq \boldsymbol{a}$. It now suffices to show that these are the only one-one degrees $\leqq \boldsymbol{a}$ and that they are all different. Let $\boldsymbol{b}$ be any one-one degree $\leqq \boldsymbol{a}$ then there exists some r.e. set $B$ such that $\Psi(B)=\boldsymbol{b}$. Now

$$
\Psi(B)=\Psi\left(B \cap R_{0}\right) \oplus \Psi\left(B \cap R_{1}\right),
$$

thus to prove that the degrees listed are the only ones $\leqq \boldsymbol{a}$ it is sufficient for $j=0,1$ to show that $\Psi\left(B \cap R_{j}\right)$ is one of $\boldsymbol{a}_{j}, \boldsymbol{m}, \mathbf{0}$. Now $\Psi$ does not distinguish between r.e. sets whose symmetric difference is finite, hence because $M$ is maximal we may suppose that $B \cap R_{j} \subseteq$ $S_{j}$ or $\left(B \cap R_{j}\right) \cup S_{j}=R_{j}$. We now consider three cases which exhaust all the possibilities. Let $\mathscr{C}_{j}=\{\{2 x+j \mid x \in X\} \mid X \in \mathscr{C}\}$.

Case 1. $B \cap R_{j} \in \mathscr{L}_{j}$. Then $B \cap R_{j} \cap A$ is finite, and so

$$
\Psi\left(B \cap R_{j}\right)=\mathbf{0} \text {. }
$$

Case 2. $B \cap R_{j} \in \mathscr{K}\left(S_{j}\right)-\mathscr{M}_{j}$. Then there exists $W$ in $\mathscr{M}_{j}$ such that $\left(B \cap R_{j}\right) \cup W=S_{j}$. Since $\mathscr{K}$ is separated there exist disjoint r.e. sets $T, U$ included in $B \cap R_{j}, W$ respectively such that $T \cup U=S_{j}$. Now $\Psi\left(S_{j}\right)=\Psi(T) \oplus \Psi(U)$. But $\Psi(W)=\mathbf{0}$, whence $\Psi(U)=\mathbf{0}$. Thus 
$\Psi(T)=\Psi\left(S_{j}\right)=\boldsymbol{m}$. Since $T \subseteq B \cap R_{j} \subseteq S_{j}$, we also have $\Psi\left(B \cap R_{j}\right)=\boldsymbol{m}$.

Case 3. $\left(B \cap R_{j}\right) \cup S_{j}=R_{j}$. Then since $\mathscr{\Upsilon}$ is separated there exist recursive sets $T, U$ included in $B \cap R_{j}, S_{j}$ respectively such that $T \cup U=R_{j}$. Since $\mathscr{L}(M) \subseteq \mathscr{l}$, we have $U \in \mathscr{C}_{j}$ whence $\Psi(U)=\mathbf{0}$. Thus $\Psi(T)=\Psi\left(R_{j}\right)=\boldsymbol{a}_{j}$. Therefore $\Psi\left(B \cap R_{j}\right)=\boldsymbol{a}_{j}$.

This completes the proof that each one-one degree $\leqq \boldsymbol{a}$ is one of the eight listed. We now turn to the task of showing that all eight are distinct. It is easy to show that no cylinder $>0$ can be represented by an immune or coimmune set. But $A \cap S_{j}$ is immune since otherwise $A \cap S_{j}$ would have an infinite recursive subset which is impossible. Hence $\boldsymbol{m}=\Psi\left(S_{j}\right)$ is represented by an immune set, whence $\boldsymbol{m} \oplus \boldsymbol{m} \neq \boldsymbol{m}$. Suppose for reductio ad absurdum that $\boldsymbol{a}_{0} \leqq \boldsymbol{a}_{1}$ then there exists a one-one partial recursive function $\rho$ such that $\operatorname{dom} \rho \subseteq R_{0}$, rng $\rho \subseteq R_{1}$, such that $\operatorname{dom} \rho$ and $R_{0} \cap(A \cup \operatorname{dom} \rho)$ are both recursive, and such that for all $x$ in $\operatorname{dom} \rho$

$$
x \in A \Leftrightarrow \rho(x) \in A .
$$

It is easy to see that $R_{0} \cap(\operatorname{dom} \rho)^{\prime} \cap A$ is finite since $A$ is immune. Therefore $(\operatorname{dom} \rho)^{\prime}$ cannot contain all but a finite number of members of $R_{0}-S_{0}$, therefore $\operatorname{dom} \rho$ does contain all but a finite number of members of $R_{0}-S_{0}$. Now $\rho\left(R_{0}-S_{0}\right)$ is clearly cohesive and so either $S_{1} \cap \rho\left(R_{0}-S_{0}\right)$ is finite or $\left(R_{1}-S_{1}\right) \cap \rho\left(R_{0}-S_{0}\right)$ is finite.

Case 1. $S_{1} \cap \rho\left(R_{0}-S_{0}\right)$ is finite. Define the one-one partial recursive function $\sigma$ by

$$
\sigma(x)=y \Leftrightarrow \rho(2 x)=2 y+1 .
$$

Then $\sigma\left(M^{\prime}\right) \cap M$ is finite. Write $x \sim y$ if either $x=y$, or $x$ can be obtained by iterating $\sigma$ on $y$, or $y$ can be obtained by iterating $\sigma$ on $x$. Since $\sigma$ is one-one, $\sim$ is an equivalence relation on $N$. Consider the equivalence classes intersecting $M^{\prime}$; none of them can have infinite intersection with $M^{\prime}$ because this would mean that $M^{\prime}$ had an infinite r.e. subset. Thus there are an infinite number of equivalence classes intersecting $M^{\prime}$. Since $\sigma\left(M^{\prime}\right) \cap M$ is finite and $M$ is maximal, $M^{\prime}-\sigma\left(M^{\prime}\right)$ is also finite. Hence only a finite number of equivalence classes intersect both $M$ and $M^{\prime}$. Since $P_{0}, P_{1}$ are disjoint it follows from (3) that $\sigma(x) \neq x$ for infinitely many $x$ in $M^{\prime}$. Hence there exist infinitely many equivalence classes $\subseteq M^{\prime}$ of cardinality $>1$. We now have a contradiction, because we can construct two disjoint r.e. sets each of which intersects all the finite equivalence classes of cardinality $>1$, and hence each of which has infinite intersection with $M^{\prime}$. 
Case 2. $\rho\left(R_{0}-S_{0}\right) \cap\left(R_{1}-S_{1}\right)$ is finite. By Theorem 3 of [2] it follows that $R_{1}-\rho\left(R_{0}-S_{0}\right)$ is r.e. and hence there exists a recursive set $T \supseteqq \rho\left(R_{0}-S_{0}\right)$ such that $T-S_{1}$ is finite. Now $T \cap S_{1}$ being recursive is in $\mathscr{C l}_{1}$ and so has only a finite intersection with $A$. Hence $\rho\left(R_{0}-S_{0}\right)$ has only a finite intersection with $A$. This contradicts (3), because $\rho$ is one-one and $R_{0}-S_{0}$ has infinite intersection with $A$.

We have now shown that $\boldsymbol{a}_{0} \not \boldsymbol{a}_{1}$; similarly $\boldsymbol{a}_{1} \$ \boldsymbol{a}_{0}$. It only remains to show that $\boldsymbol{a}_{j} / \boldsymbol{m}=1$ for $j=0,1$. Suppose for reductio ad absurdum that $\boldsymbol{a}_{j} / \boldsymbol{m} \neq 1$, then $\boldsymbol{a}_{j}=\boldsymbol{m} \oplus \boldsymbol{m}$, because we have shown above that the only one-one degrees $<\boldsymbol{a}_{j}$ are $\mathbf{0}$ and $\boldsymbol{m}$. Since $\boldsymbol{a}_{j}=$ $\Psi\left(R_{j}\right)=\boldsymbol{m} \oplus \boldsymbol{m}$ there exist disjoint recursive sets $T, U$ whose union is $R_{j}$ such that $\Psi(T)=\Psi(U)=\boldsymbol{m}$. One of $T, U$ has only finite intersection with $R_{j}-S_{j}$. Thus we may suppose $T \subseteq S_{j}$, whence $T \in \mathscr{C}_{j}$, which means that $T \cap A$ is finite. Therefore $\Psi(T)=0$, again a contradiction. Using the results of $\S 2$ it now follows easily that the initial segment of one-one degrees $\leqq \boldsymbol{a}$ is isomorphic to the nondistributive lattice depicted above.

The use of Theorem 3 of [2] may be eliminated if we construct $A \cap\left(R_{0}-S_{0}\right), A \cap\left(R_{1}-S_{1}\right)$ with the immediate intention that $\boldsymbol{a}_{0}, \boldsymbol{a}_{1}$ should be incomparable and both $>\boldsymbol{m}$.

4. Recursively enumerable initial segments. In this section we prove a theorem about r.e. one-one degrees and deduce that all r.e. finite initial segments are distributive. The theorem we shall prove is of the same genre as Theorem 3 of [2] quoted above.

THEOREM 6. Let $\boldsymbol{a}, \boldsymbol{b}$ be indecomposable r.e. one-one degrees such that $\boldsymbol{b}<\boldsymbol{a}$, then $\boldsymbol{a}=\boldsymbol{a} \oplus \boldsymbol{b}$.

Proof. Let $A$ be a representative of $\boldsymbol{a}$. Let $\Psi$ be the map of the r.e. sets onto the one-one degrees $\leqq a$ defined in the last section. It is clear that $\Psi(W)=\Psi(W \cup A)$ for every r.e. set $W$. Let $\left\{\varphi_{i}\right\}$ be a recursive enumeration of all p.r. functions of one argument. Let $\left\{\varphi_{x, y}\right\}$ be an s.r.e. double sequence of finite functions such that for each $x, \varphi_{x, 0} \subseteq \varphi_{x, 1} \subseteq \cdots$ and $\lim _{y} \varphi_{x, y}=\varphi_{x}$. Let $B$ be an infinite r.e. set such that $B \supseteqq A$ and $\Psi(B)=\boldsymbol{b}$. Let $\beta$ be a one-one recursive function enumerating $B$. We shall effectively enumerate in steps $0,1,2, \cdots$ disjoint r.e. sets $B^{0}, B^{1}$ whose union is $B$. For $j=0,1$ let $B_{s}^{j}$ be the finite set of numbers enumerated in $B_{j}$ before step $s$. While enumerating $B^{0}, B^{1}$ we simultaneously enumerate $A$. Let $A_{s}$ be the finite set of numbers enumerated in $A$ before step $s$. Let $P$ be the set of all pairs $(e, j)$ of natural numbers with $j \leqq 1$. We will order $P$ according to 


$$
(e, j)<(f, k) \Leftrightarrow[e<f \cdot \mathbf{V} \cdot e=f \text { and } j<k] .
$$

In order to describe step $s$ we need two auxiliary functions:

$$
\gamma_{s}^{j}(e)=\sup \left\{x \mid x=e \bigvee(y)_{y<x}\left[y \in B_{s}^{j} \rightarrow: \varphi_{e, s}(y)\right.\right.
$$

is defined $\left.\left.\cdot \& \cdot \varphi_{e, s}(y) \in B_{s}^{1-j} \cdot \& \cdot y \in A_{s} \Leftrightarrow \varphi_{e, s}(y) \in A_{s}\right]\right\}$,

$$
\delta_{s}^{j}(e)=\sup \left[\gamma_{x}^{k}(y) \mid x \leqq s \quad \& \quad(y, k) \leqq(e, j) \quad \& \quad(y, k) \in P\right\}
$$

each defined only for $(e, j)$ in $P$. The construction can now be stated very easily. In step $s$ let $(e, j)$ be the least pair in $P$ such that $\beta(s)<\delta_{s}^{j}(e)$. Enumerate $\beta(s)$ in $B^{j}$.

To prove the theorem notice that

$$
\boldsymbol{b}=\Psi(B)=\Psi\left(B^{0}\right) \oplus \Psi\left(B^{1}\right) .
$$

Since $\boldsymbol{b}$ is indecomposable, for either $j=0$ or $j=1$ we have $\Psi\left(B^{j}\right) \leqq$ $\Psi\left(B^{1-j}\right)$. Thus there exists a partial recursive one-one function $\varphi_{e}$ such that $B^{j}-\operatorname{dom} \varphi_{e}, B^{j}-\left(A \cup \operatorname{dom} \varphi_{e}\right)$ are both r.e., such that $\operatorname{dom} \varphi_{e} \subseteq B^{j}$, $\operatorname{rng} \varphi_{e} \subseteq B^{1-j}$, and

$$
y \in A \Leftrightarrow \varphi_{e}(y) \in A
$$

for all $y$ in $\operatorname{dom} \varphi_{e}$. Now $A \cap B^{1-j}$ is obviously infinite and r.e., thus we may suppose that $B^{j}$-dom $\varphi_{e} \subseteq A^{\prime}$. Further, if $B^{1-j}-A$ has an infinite recursive subset, we may suppose that $B^{j}=\operatorname{dom} \varphi_{e}$. However, if $B^{1-j}-A$ is immune, we may suppose that $B$ was chosen so that $B-A$ is since $\Psi\left(B^{1-j}\right)=b$. Thus in any case we may suppose that $B^{j}-\operatorname{dom} \varphi_{e}$ is finite. By a suitable adjustment of $\varphi_{e}$ we can make $\operatorname{dom} \varphi_{e}=B^{j}$ unless $\Psi\left(B^{j}\right)=\Psi\left(B^{1-j}\right)$. However, if $\Psi\left(B^{j}\right)=\Psi\left(B^{1-j}\right), \boldsymbol{b}$ is a cylinder and the theorem is immediate. Thus we shall suppose that $\operatorname{dom} \varphi_{e}=B^{j}$. Clearly, $\lim _{x} \gamma_{x}(e, j)=\infty$ whence $\lim _{x} \delta_{x}(e, j)=\infty$. Let $(e, j)$ now be the least pair in $P$ such that $\lim _{x} \delta_{x}(e, j)=\infty$, then $\lim _{x} \gamma_{x}(e, j)=\infty$ and $\Psi\left(B^{j}\right) \leqq \Psi\left(B^{1-j}\right)=b$. For each pair $(f, k)$ in $P$ which is $<(e, j), \lim _{x} \delta_{x}(f, k)$ is finite because $\delta_{s}(f, k)$ is increasing with $s$. Let

$$
m=\sup \left\{\delta_{x}(f, k) \mid x \geqq 0 \quad \& \quad(f, k) \in P \quad \& \quad(f, k)<(e, j)\right\} .
$$

At any step $s$ where $\beta(s) \geqq m$, we can have $\beta(s)$ enumerated in $B^{1-j}$ only if $\delta_{s}(e, j) \leqq \beta(s)$. But $\delta_{s}(e, j)$ increases with $s$ and $\lim _{x} \delta_{x}(e, j)=\infty$, therefore $B^{1-j}$ is recursive. Now

$$
\boldsymbol{a}=\Psi(N)=\Psi\left(B^{1-j}\right) \oplus \psi\left(\left(B^{1-j}\right)^{\prime}\right) .
$$

But $\boldsymbol{a}$ is indecomposable and $\Psi\left(B^{1-j}\right)=\boldsymbol{b}<\boldsymbol{a}$, whence $\Psi\left(\left(B^{1-j}\right)^{\prime}\right)=\boldsymbol{a}$. The last equation is now $\boldsymbol{a}=\boldsymbol{b} \oplus \boldsymbol{a}$ which proves the theorem. 
THEOREM 7. Let a be an r.e. one-one degree which has only a finite number of predecessors then the one-one degrees $\leqq$ a form $a$ distributive lattice.

Proof. Let $\boldsymbol{b}, \boldsymbol{c}, \boldsymbol{a}^{*}$ be one-one degrees $\leqq \boldsymbol{a}$ such that one of $\boldsymbol{b} / \boldsymbol{a}^{*}$, $\boldsymbol{c} / \boldsymbol{a}^{*}$ is finite. Then $\min \left(\boldsymbol{b} / \boldsymbol{a}^{*}, \boldsymbol{c} / \boldsymbol{a}^{*}\right) \boldsymbol{a}^{*}$ is $\leqq$ both $\boldsymbol{b}$ and $\boldsymbol{c}$. Hence

$$
\boldsymbol{b} \cap \boldsymbol{c} / \boldsymbol{a}^{*}=\min \left(\boldsymbol{b} / \boldsymbol{a}^{*}, \boldsymbol{c} / \boldsymbol{a}^{*}\right) .
$$

It is clear that we can relax the condition that one of $\boldsymbol{b} / \boldsymbol{a}^{*}, \boldsymbol{c} / \boldsymbol{a}^{*}$ be finite. Now let $\boldsymbol{a}^{*}$ be a maximal indecomposable degree $\leqq \boldsymbol{a}^{*}$. Then in the proof of Theorem 4 we may choose $a_{1}=a^{*}$ and so from the construction of $\boldsymbol{b} \cup \boldsymbol{c}$ in that proof

$$
\boldsymbol{b} \cup \boldsymbol{c} / \boldsymbol{a}^{*}=\max \left(\boldsymbol{b} / \boldsymbol{a}^{*}, \boldsymbol{c} / \boldsymbol{a}^{*}\right) .
$$

It is clear that (6) also holds when $\boldsymbol{a}^{*}$ is a cylinder.

Now let $\boldsymbol{x}, \boldsymbol{y}, \boldsymbol{z}$ be any r.e. one-one degrees $\leqq \boldsymbol{a}$, to prove the theorem it is sufficient to prove

$$
(\boldsymbol{x} \cup \boldsymbol{y}) \cap z \leqq(\boldsymbol{x} \cap z) \cup(\boldsymbol{y} \cap z) .
$$

From Lemma 2 it is therefore sufficient to show that

$$
\{(\boldsymbol{x} \cup \boldsymbol{y}) \cap z\} / \boldsymbol{a}^{*} \leqq\left\{(\boldsymbol{x} \cap z) \cup(\boldsymbol{y} \cap z\} / \boldsymbol{a}^{*}\right.
$$

for every indecomposable degree $\boldsymbol{a}^{*} \leqq \boldsymbol{a}$. From Theorem 6 any such $\boldsymbol{a}^{*}$ is either a maximal indecomposable degree $\leqq \boldsymbol{a}$ or is a cylinder, thus (5) and (6) are applicable. Using (5) and (6) this reduces to showing that

$$
\min \left(\max \left(\boldsymbol{x} / \boldsymbol{a}^{*}, \boldsymbol{y} / \boldsymbol{a}^{*}\right), z / \boldsymbol{a}^{*}\right) \leqq \max \left(\min \left(\boldsymbol{x} / \boldsymbol{a}^{*}, z / \boldsymbol{a}^{*}\right),\right.
$$

$\left.\min \left(\boldsymbol{y} / \boldsymbol{a}^{*}, \boldsymbol{z} / \boldsymbol{a}^{*}\right)\right)$.

However, it is easy to verify that equality obtains in the last line using only the definitions of $\max$ and min. This completes the proof of the theorem.

5. Conclusion. Above we have hardly touched upon the general question of characterizing arbitrary initial segments of the one-one degrees. A certain amount of information can be obtained by studying the map $\Psi$ introducted in the proof of Lemma 2. However, there is a serious obstacle to a complete solution of the problem. Suppose that we are considering the one-one degrees $\leqq \boldsymbol{a}$ where $\boldsymbol{a}$ is a cylinder. If there is a noncylinder $\boldsymbol{b} \leqq \boldsymbol{a}$ then from the work of Young in [7] there exists a collection of one-one degrees $\leqq \boldsymbol{a}$, and in the same manyone degree as $\boldsymbol{b}$, which has the order type of the rationals. Thus it 
seems that the approach to initial segments via finite substructures which has been successful for the many-one degrees in [3], is doomed to failure in the case of one-one degrees.

Our final comment concerns the relation between the notion of one-one degree used in this paper and the usual notion. Let $\boldsymbol{a}$ be the one-one degree of a simple set such that the order types of the initial segments of one-one degrees $\leqq \boldsymbol{a}$, under our definition and the usual definition, are $\theta$ and $\theta^{*}$ respectively. From Theorem 1 of [6] it is easy to see that

$$
\theta^{*}=\omega \oplus\left\{(\theta-\{1\}) \circ\left(\omega^{*} \oplus \omega\right)\right\} \oplus \omega^{*},
$$

where $\theta-\{1\}$ is the order type obtained from $\theta$ by deleting its greatest member, where $\oplus$ and ${ }^{\circ}$ denote ordinal sum, and product respectively, where $\omega$ is the order type of the natural numbers, and where $\omega^{*}$ is the converse of $\omega$.

\section{REFERENCES}

1. A. H. Lachlan, Distributive initial segments of the degrees of unsolvability, Z. Math. Logik Grundlagen Math. (to appear)

2. A. H. Lachlan, The lattice of recursively enumerable sets, Trans. Amer. Math. Soc. 130 (1968), 1-37.

3. Initial segments of many-one degrees, (to appear)

4. J. Myhill, Creative sets, Z. Math. Logik Grundlagen Math. 1 (1956), 97-108.

5. - Recursive digraphs, splinters, and cylinders, Math. Ann. 138 (1959), 211218.

6. P. R. Young, On reducibility by recursive functions, Proc. Amer. Math. Soc. 15 (1964), 889-892.

7. - Linear orderings under one-one reducibility, J. Symbolic Logic 31 (1966), $70-85$.

Received August 30, 1967. 


\section{PACIFIC JOURNAL OF MATHEMATICS}

\section{EDITORS}

H. ROYDEN
Stanford University
Stanford, California

\section{R. R. PHELPS}

University of Washington

Seattle, Washington 98105
J. DUGUNDJI

Department of Mathematics

University of Southern California

Los Angeles, California 90007

\section{RICHARD ARENS}

University of California

Los Angeles, California 90024

\section{ASSOCIATE EDITORS}

E. F. BeCKenbaCh
B. H. NEUMANN

F. WOLF
K. YoshidA

\section{SUPPORTING INSTITUTIONS}

\author{
UNIVERSITY OF BRITISH COLUMBIA \\ CALIFORNIA INSTITUTE OF TECHNOLOGY \\ UNIVERSITY OF CALIFORNIA \\ MONTANA STATE UNIVERSITY \\ UNIVERSITY OF NEVADA \\ NEW MEXICO STATE UNIVERSITY \\ OREGON STATE UNIVERSITY \\ UNIVERSITY OF OREGON \\ OSAKA UNIVERSITY \\ UNIVERSITY OF SOUTHERN CALIFORNIA
}

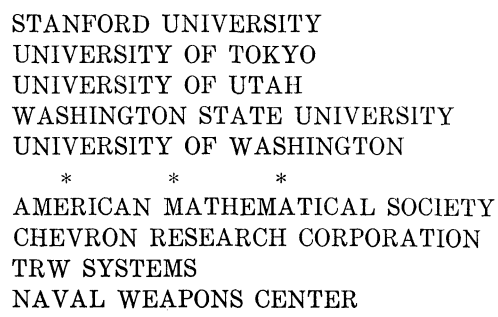

The Supporting Institutions listed above contribute to the cost of publication of this Journal, but they are not owners or publishers and have no responsibility for its content or policies.

Mathematical papers intended for publication in the Pacific Journal of Mathematics should be in typed form or offset-reproduced, double spaced with large margins. Underline Greek letters in red, German in green, and script in blue. The first paragraph or two must be capable of being used separately as a synopsis of the entire paper. It should not contain references to the bibliography. Manuscripts, in duplicate if possible, may be sent to any one of the four editors. Please classify according to the scheme of Math. Rev. 36, 1539-1546. All other communications to the editors should be addressed to the managing editor, Richard Arens, University of California, Los Angeles, California, 90024.

50 reprints are provided free for each article; additional copies may be obtained at cost in multiples of 50 .

The Pacific Journal of Mathematics is published monthly. Effective with Volume 16 the price per volume (3 numbers) is $\$ 8.00$; single issues, $\$ 3.00$. Special price for current issues to individual faculty members of supporting institutions and to individual members of the American Mathematical Society: $\$ 4.00$ per volume; single issues $\$ 1.50$. Back numbers are available.

Subscriptions, orders for back numbers, and changes of address should be sent to Pacific Journal of Mathematics, 103 Highland Boulevard, Berkeley, California, 94708.

PUBLISHED BY PACIFIC JOURNAL OF MATHEMATICS, A NON-PROFIT CORPORATION

Printed at Kokusai Bunken Insatsusha (International Academic Printing Co., Ltd.), 7-17, Fujimi 2-chome, Chiyoda-ku, Tokyo, Japan. 


\section{Pacific Journal of Mathematics \\ Vol. 29, No. $2 \quad$ June, 1969}

Bruce Langworthy Chalmers, On boundary behavior of the Bergman kernel function and related domain functionals ................... 243

William Eugene Coppage, Peirce decomposition in simple Lie-admissible power-associative rings .............................. 251

Edwin Duda, Compactness of mappings...................... 259

Earl F. Ecklund Jr., On prime divisors of the binomial coefficient......... 267

Don E. Edmondson, A modular topological lattice ............... 271

Phillip Alan Griffith, A note on a theorem of Hill ................... 279

Marcel Herzog, On finite groups with independent cyclic Sylow

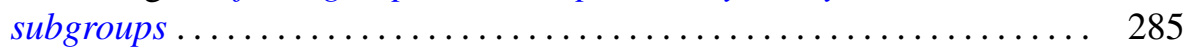

James A. Huckaba, Extensions of pseudo-valuations................. 295

S. A. Huq, Semivarieties and subfunctors of the identity functor ........ 303

I. Martin (Irving) Isaacs and Donald Steven Passman, Finite groups with small character degrees and large prime divisors. II ............ 311

Carl Kallina, A Green's function approach to perturbations of periodic

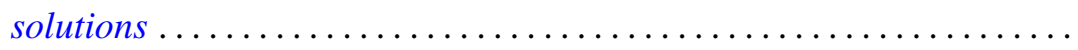

$\mathrm{Al}$ (Allen Frederick) Kelley, Jr., Analytic two-dimensional subcenter manifolds for systems with an integral ....................

Alistair H. Lachlan, Initial segments of one-one degrees ............ 351

Marion-Josephine Lim, Rank k Grassmann products ............. 367

Raymond J. McGivney and William Henry Ruckle, Multiplier algebras of

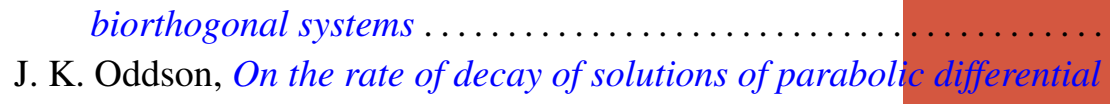

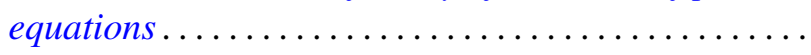

Helmut R. Salzmann, Geometries on surfaces ........... .

Annemarie Schlette, Artinian, almost abelian groups and their groups of automorphisms ............................

Edgar Lee Stout, Additional results on modules over polydisc algebras ...

Lajos Tamássy, A characteristic property of the sphere . .

Mark Lawrence Teply, Some aspects of Goldie's torsion theory. ...

Freddie Eugene Tidmore, Extremal structure of star-shaped sets ...

461

Leon Jarome Weill, Unconditional and shrinking bases in locally convex spaces... 